\section{Case Reports in Dermatology}

This article is licensed under the Creative Commons Attribution-NonCommercial 4.0 International License (CC BY-NC) (http://www.karger.com/Services/OpenAccessLicense) Usage and distribution for commercial purposes requires written permission.

\title{
Itolizumab - A New Biologic for Management of Psoriasis and Psoriatic Arthritis
}

\author{
Ganesh Pai Anusha H. Pai \\ Derma-Care Skin and Cosmetology Centre, Mangalore, India
}

\section{Keywords}

Psoriasis $\cdot$ Itolizumab $\cdot$ Psoriatic arthritis

\begin{abstract}
Psoriasis is a chronic, relapsing, inflammatory, immune-mediated systemic disease with mainly skin and joint manifestations. The available treatment options to cure psoriasis include topical therapy, phototherapy, and biological therapy. Biological therapy has become a promising option due to the rapid action and less adverse effects associated with its use. The newly developed biologic itolizumab is a humanized recombinant anti-CD6 monoclonal antibody of IgG1 isotype that binds to domain 1 of CD6, thereby immunomodulating human lymphocytes without interfering with the binding of CD6 to the activated leukocyte-cell adhesion molecule. In this case series, a total of 5 patients with chronic plaque psoriasis were treated with itolizumab. They exhibited a rapid PASI 75 response after 4 doses of itolizumab infusion. The patients were poor responders to methotrexate and/or cyclosporine therapy for a long time. All infusions were well tolerated by all patients with no adverse reactions or infections during the treatment period. Itolizumab can be a good option for management of psoriasis and psoriatic arthritis.

(C) 2017 The Author(s)

Published by S. Karger AG, Basel
\end{abstract}




\section{Introduction}

Psoriasis is a chronic, relapsing, inflammatory, immune-mediated systemic disease with mainly skin and joint manifestations [1]. It is characterized by skin lesions containing red, scaly, and erythematous patches, and papules which normally itch. The prevalence of the disease is $2-3 \%$ of a population, and it equally affects both genders. The etiology of the disease is not completely understood. It is not only a skin disease; it also affects the quality of life of the patient [2]. No complete cure for psoriasis is available, but several treatments can help to control symptoms including topical therapy, phototherapy, and biological therapy. Biological therapy is now emerging as a promising option for the treatment of psoriasis due to its rapid action, less toxicity to the liver, kidney, and bone marrow, and it is nonteratogenic [3]. Biological therapy acts by specifically targeting T cells and inflammatory cytokines. A new molecule, itolizumab, developed and used in India, is a humanized recombinant antiCD6 monoclonal antibody of IgG1 isotype that binds to domain 1 of CD6, thereby immunomodulating human lymphocytes without interfering with the binding of CD6 to the activated leukocyte-cell adhesion molecule [4]. The objective of the treatment was rapid reduction and control of complicated and extensive psoriasis with the use of itolizumab.

\section{Case Presentation}

A total of 5 patients with a history of prolonged cycles of methotrexate and/or cyclosporine therapy with poor response visited our clinic. Four out of them had chronic plaque psoriasis, and 1 patient had psoriasis along with psoriatic arthropathy. All patients were treated with itolizumab (Alzumab). The regimen was bimonthly cycles administered intravenously in $0.9 \%$ normal saline at a dose of $1.6 \mathrm{mg} / \mathrm{kg}$ for 3 months followed by maintenance with monthly cycles for 3 months.

\section{Patient 1}

The patient had multiple psoriatic plaques on his body and scalp for which he took methotrexate tablets and topical treatment like moisturizers, calcipotriol, and steroid creams. Psoriatic plaques increased in size, and new lesions appeared. They involved more than $90 \%$ of his body surface area. Subsequently, he took ayurvedic medication, but there was no improvement. The patient developed psoriatic arthropathy during which he consulted us, and he was given Alzumab injections.

\section{Patient 2}

The patient had multiple psoriatic plaques on her body, for which she took methotrexate orally along with topical treatment consisting of moisturizer and steroids for 2 years, and then she tried ayurvedic medications. New lesions appeared. Alzumab infusions were started, after which the lesions subsided. 


\section{Patient 3}

The patient had multiple psoriatic plaques on the body and the scalp. She took methotrexate for 6 months along with topical treatment consisting of moisturizer and steroids, after which Alzumab infusions were started.

\section{Patient 4}

The patient had multiple plaques on her body and scalp, for which she took etanercept injections for 2 months. But the lesions kept increasing, and Alzumab injections were started.

\section{Patient 5}

The patient was treated with oral methotrexate and topical application of moisturizers for psoriatic plaques on her body and scalp. She was treated with phototherapy for 6 months, after which the plaques resolved. New lesions started appearing after 2 years. Alzumab infusions were started.

The demographic details of psoriasis patients are depicted in Table 1. Psoriasis area and severity index (PASI) were assessed at baseline and after the fourth dose of itolizumab. Significant changes in the PASI score observed during the treatment of itolizumab are presented in Table 2.

The entire group of patients showed significant improvement in PASI scores before the onset of the third dose of itolizumab. Baseline PASI scores of all those patients were 52, 24.2, $45,43.2$, and 48.2 (serially) and on completion of the fourth dose; PASI scores decreased to $5.5,4.2,6.5,5.3$, and 5.5, respectively. Significantly improvement in the case of psoriatic arthropathy was also reported during the induction dosing regimen of itolizumab. All infusions were well tolerated by all patients with no adverse reactions or infections during the treatment period.

\section{Discussion}

Psoriasis is a chronic, long-lasting disease of the skin, and often requires lifelong treatment. The revolution in the treatment of psoriasis disease occurred after the introduction of biological therapy. Biologics are highly specific proteins which act on the immune or genetic mediator of a pathophysiological process of the disease [5]. In our study, all patients exhibited improvement in the PASI score during the treatment period. All patients attained a greater than PASI 75 response on completion of the fourth dose of itolizumab (Fig. 1). PASI 75, or a decline in the baseline PASI score of greater than $75 \%$, is the standard used by the FDA to evaluate the efficacy of an antipsoriatic drug [6].

Itolizumab induced a rapid PASI75 response in psoriasis patients, who were poor responders to methotrexate and/or cyclosporine therapy for a long time. In a phase 3 study, Krupashankar et al. [7] reported PASI 75 response at week 12 after treatment of itolizumab in chronic plaque psoriasis patients. No case of any adverse event was reported during the study. Itolizumab was well tolerated and found to be safe. The treatment cost of itolizumab is lower as compared to other biologics. The results of the study indicate that itolizumab can be a good option for management of psoriasis and psoriatic arthritis. 


\section{Conclusion}

Itolizumab is a novel, safe, and efficacious therapy for the management of psoriasis offering a ray of hope for those who are severely and chronically affected by this disease. It is also much more affordable than other currently available monoclonal antibodies and has comparable efficacy and safety.

\section{Statement of Ethics}

The authors have no ethical conflicts to disclose.

\section{Disclosure Statement}

The authors declare no conflicts of interest.

\section{References}

1 Winterfield LS, Menter A, Gordon K, Gottlieb A: Psoriasis treatment: current and emerging directed therapies. Ann Rheum Dis 2005; 64:ii87-ii90.

-2 Lazaro EA, Lopez-Requena A, Fuentes D, et al: Immunological and histological evaluation of clinical samples from psoriasis patients treated with anti-CD6 itolizumab. MAbs 2014;6:782-792.

3 Menter A, Korman NJ, Elmets CA, et al: Guidelines of care for the management of psoriasis and psoriatic arthritis. J Am Acad Dermatol 2009;61:451-485.

-4 Menon R, David BG: Itolizumab - a humanized anti-CD6 monoclonal antibody with a better side effects profile for the treatment of psoriasis. Clin CosmetInvestig Dermatol 2015;8:215-222.

5 Sivamani RK, Correa G, Ono Y, et al: Biological therapy of psoriasis. Indian J Dermatol 2010:55:161-170.

6 Koo J, Khera P: Update on the mechanisms and efficacy of biological therapies for psoriasis. J Dermatol Sci 2005;38:75-87.

7 Krupashankar DS, Dogra S, Kura M, et al: Efficacy and safety of itolizumab, a novel anti-CD6 monoclonal antibody, in patients with moderate to severe chronic plaque psoriasis: results of a double-blind, randomized, placebo-controlled, phase-III study. J Am Acad Dermatol 2014;71:484-492.

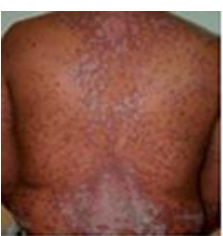

Before

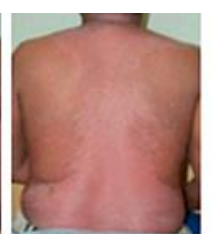

After

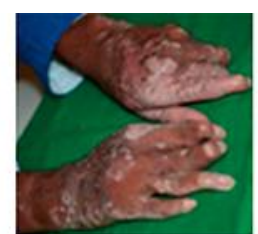

Before

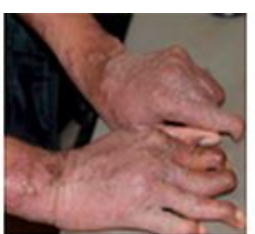

After

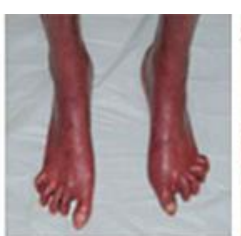

Before

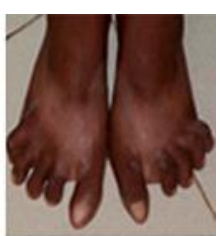

After

Fig. 1. Effect of itolizumab treatment on psoriasis. 
Table 1. Demographic details of psoriasis patients

\begin{tabular}{llllll}
\hline Demographic detail & Patient 1 & Patient 2 & Patient 3 & Patient 4 & Patient 5 \\
\hline Age, years & 28 & 26 & 50 & 22 & 57 \\
Sex & Male & Female & Female & Female & Female \\
Weight, kg & 60 & 72 & 55 & 58 & 56 \\
\hline
\end{tabular}

Table 2. Effect of itolizumab treatment on the PASI score of psoriasis patients

\begin{tabular}{lll}
\hline Patient No. & \multicolumn{2}{l}{ PASI score } \\
\cline { 2 - 3 } & baseline & after completion of 4 doses \\
\hline 1 & 52 & 5.5 \\
2 & 24.2 & 4.2 \\
3 & 45 & 6.5 \\
4 & 43.2 & 5.3 \\
5 & 48.2 & 5.5 \\
\hline
\end{tabular}

